\title{
Novel Instrumental Approach to the Measurement of Total Body Water: Optothermal Detection of Heavy Water in the Blood Serum
}

\author{
JASPER ANNYAS, DANE BICANIC, * and FRANS SCHOUTEN \\ Laser Photoacoustic Laboratory, Wageningen University and Research Centre, Bomenweg 4, 6703 HD Wageningen, \\ The Netherlands (J.A., D.B.); and Department of Human Nutrition and Epidemiology, Wageningen University and Research Centre \\ Bomenweg 2, 6703 HD Wageningen, The Netherlands (F.S.)
}

\begin{abstract}
The optothermal window $(\mathrm{OW})$ in conjunction with a low-power infrared source was used at $4 \mu \mathrm{m}$ wavelength to measure the concentration of $D_{2} O$ needed to determine the total body water (TBW) in human subjects. The 30 ppmv $D_{2} O$ detection limit is comparable to that obtainable by infrared transmission (IRT) spectroscopy but can be further enhanced. Low cost and ease of sampling and operation are other advantages of the novel method.

Index Headings: Optothermal window; Infrared spectroscopy; Photothermal spectroscopy; Total body water.
\end{abstract}

\section{INTRODUCTION}

The measurement of the total body water (TBW) is one of the well-established methods to estimate the fat content of a human individual, with bioelectrical impedance analysis (BIA) and tracer dilution (TD) being the two most common in vivo approaches used. ${ }^{1}$ In BIA two electrodes are attached directly to the skin, and TBW is calculated from measured impedance. When using the TD approach, one needs to obtain a dilution factor after initial ingestion of a well-known quantity of the tracer compound. This dilution factor is calculated after concentration of a tracer in the blood has been measured at some later stage. ${ }^{2}$

Heavy water is a suitable TBW tracer since it diffuses into all body compartments and is also treated by a human body in the same way as $\mathrm{H}_{2} \mathrm{O} .{ }^{3}$ Orally administrated $10 \mathrm{~g}$ of $\mathrm{D}_{2} \mathrm{O}$ eventually results in 100 to $600 \mathrm{ppmv} \mathrm{D}_{2} \mathrm{O}$ in the blood serum. One method used to determine the concentration of $\mathrm{D}_{2} \mathrm{O}$ in biological fluids is infrared spectroscopy at a $4 \mu \mathrm{m}$ wavelength, at which the spectral contrast between $\mathrm{D}_{2} \mathrm{O}$ and water is maximal. As neat liquids, both $\mathrm{D}_{2} \mathrm{O}$ and water exhibit strong absorptions ${ }^{4}$ at $4 \mu \mathrm{m}\left(\beta_{\mathrm{H}_{2} \mathrm{O}}=144 \mathrm{~cm}^{-1}, \beta_{\mathrm{D}_{2} \mathrm{O}}=18000 \mathrm{~cm}^{-1}\right.$, where $\beta$ is the absorption coefficient per unit length), which imposes restrictions on cell pathlength when conventional infrared transmission (IRT) spectroscopy is used for quantitative analysis. Such difficulties encountered in practice when studying optically dense liquids can be partially alleviated if attenuated total reflectance (ATR) is used instead of IRT. ${ }^{5}$

The main objective of the laboratory study described here was to investigate the potential of a new method, the so-called optothermal window $(\mathrm{OW})$, as a new approach to measure $\mathrm{D}_{2} \mathrm{O}$ needed to determine TBW. The operational principle of $\mathrm{OW}$ is as follows: the periodi-

Received 23 July 1998; accepted 26 October 1998.

* Author to whom correspondence should be sent. cally modulated infrared radiation $(4 \mu \mathrm{m})$ passes through an optically transparent and thin disk that carries the test sample (Fig. 1). The heat generated in the sample by the absorption of radiation leads to the formation of thermal waves that propagate back to the disk, causing its temperature to fluctuate periodically; as a result, the disk will expand and contract periodically. The annular piezoelectric ring glued to the bottom of the disk (these two components together constitute the $\mathrm{OW}$ ) converts such dimensional changes into the electrical voltage (OW signal). The magnitude and the phase of the OW signal contain information about optical and thermal properties of the test sample. Under some experimental conditions, the magnitude of the OW signal has been shown to be directly proportional to the product of the absorption strength $\beta c$ [where $c$ is the (dimensionless) fractional concentration] and to the incident power of the excitation source. ${ }^{6,7}$

The performance of the OW method was investigated with the use of standard aqueous solutions of $\mathrm{D}_{2} \mathrm{O}(25$ to $800 \mathrm{ppmv})$ and blood serum samples $\left(\mathrm{D}_{2} \mathrm{O}\right.$ ranging from 150 to $400 \mathrm{ppmv}$ ) from human subjects. The results obtained were then compared with those obtained from same samples by means of IRT.

\section{EXPERIMENTAL}

The experimental setup (Fig. 2) used in this study comprises a thermal source, the modulator, a number of optical components, the OW device, and the electronic equipment needed to detect and process the signal. The thermal source was a CL 921 lamp from Carley Lamps, Torrance, CA, U.S.A. The chopper (2 to $75 \mathrm{~Hz}$ ) was made in-house, and the interference filter (central wavelength $4 \mu \mathrm{m}$ bandwidth at a half-maximum $0.1024 \mu \mathrm{m}$ ) was a product of FK Optical Limited, Tyne and Wear, England. Two lenses in a series were used to collect the lamp radiation and to focus it onto the OW. The planoconvex lens $\mathrm{L}_{1}$ (Melles Griot, New York, U.S.A) was made of sapphire (diameter $20 \mathrm{~mm}$, focal length $20 \mathrm{~mm}$ ) and AR/AR coated for $4 \mu \mathrm{m}$. The $\mathrm{CaF}_{2}$ biconvex lens $\mathrm{L}_{2}$ (Janos Technology Inc., Vermont, U.S.A.) was $12.7 \mathrm{~mm}$ in diameter and had a focal length of $19 \mathrm{~mm}$.

The OW manufactured by Varilab AB, Huddinge, Sweden was a $0.30 \mathrm{~mm}$ thick and uncoated $14 \mathrm{~mm}$ diameter sapphire disk (density $\rho=3.98 \times 10^{3} \mathrm{~kg} / \mathrm{m}^{3}$; specific heat $c_{\mathrm{\rho}}=7.52 \times 10^{2} \mathrm{~J} / \mathrm{kgK}$; thermal conductivity $\kappa=32.8 \mathrm{~W} / \mathrm{mK}$ ) with the annular piezoelectric ring (lead zirconate titanate PZT) glued to its rear side, leaving a 


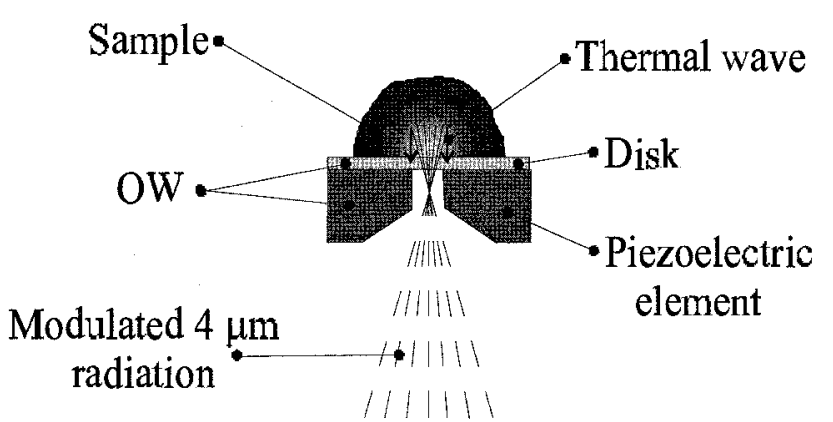

FIG. 1. The operating principle of the OW (see text for detailed explanation). The lighter region indicates the part of the sample that is being heated periodically.

clear area (diameter approximately $3 \mathrm{~mm}$ ) for passage of the incident radiation. The source, the modulator, all optical components, and the OW were assembled together (their symmetry and optical axes coincided in space) into a single unit that itself was mounted on an L-shaped platform as shown in Fig. 3.

The 1 in. diameter CL 921 lamp has an integrated ellipsoidal reflector and the internal collimating sapphire lens, providing a nearly parallel beam of radiation. In order to achieve stable emission, the lamp was accommodated within a coaxial cooling jacket (see Fig. 3) connected to a regulated $\left(20{ }^{\circ} \mathrm{C}\right)$ thermostat bath. When operated at $5 \mathrm{~V}$ and $1.5 \mathrm{~A}$, the power (measured directly in front of the lamp) emitted by the lamp over all wavelengths is $1.65 \mathrm{~W}$ The insertion of a $4 \mu \mathrm{m}$ interference filter causes this power to drop down to $70 \mathrm{~mW}$. With both lenses $L_{1}$ and $L_{2}$ and the empty $O W$ all being in the radiation path, the power available for excitation of the sample decreased further to $2.7 \mathrm{~mW}$. A single layer of a thin aluminum foil lined along the inner wall of PZT ring prevented the forward-scattered incident radiation from directly hitting the PZT (which may otherwise cause the generation of unwanted background signals) without simultaneously affecting the amount of power transmitted through the disk.

In a typical OW experiment, test sample is pipetted to cover a clear area of the OW; one droplet of $300 \mu \mathrm{L}$ produces a layer of liquid approximately $3 \mathrm{~mm}$ thick. Although for a 400 ppmv $\mathrm{D}_{2} \mathrm{O}$ test sample (which is a typical concentration of interest here) the optical penetration depth $1 / \beta$ equals $66 \mu \mathrm{m}$, meaningful quantitative measurements can still be performed, because the actual physical thickness (here $3 \mathrm{~mm}$ ) is of no concern in the OW measurement ${ }^{6}$ as long as it exceeds one thermal diffusion length (for our samples about $95 \mu \mathrm{m}$ at $5 \mathrm{~Hz}$ ). An aluminum cap (see Fig. 3) served to reduce disturbing effects of the laboratory acoustic noise and the air draught.

The signal from the OW was fed into a home-made current-to-voltage preamplifier, the output of which was connected to the signal input of a two-phase lock-in amplifier SR830 (Stanford Research Systems). The synchronous detection took place at $5 \mathrm{~Hz}$; the resulting lock-in output signal was stored on a computer.

The series of standard $\mathrm{D}_{2} \mathrm{O}$ aqueous solutions were prepared with 99.9 atom \% D manufactured by Isotec Inc., Ohio, U.S.A. Lock-in signals obtained from aqueous concentrations containing $25,50,75,100,200,400,600$,

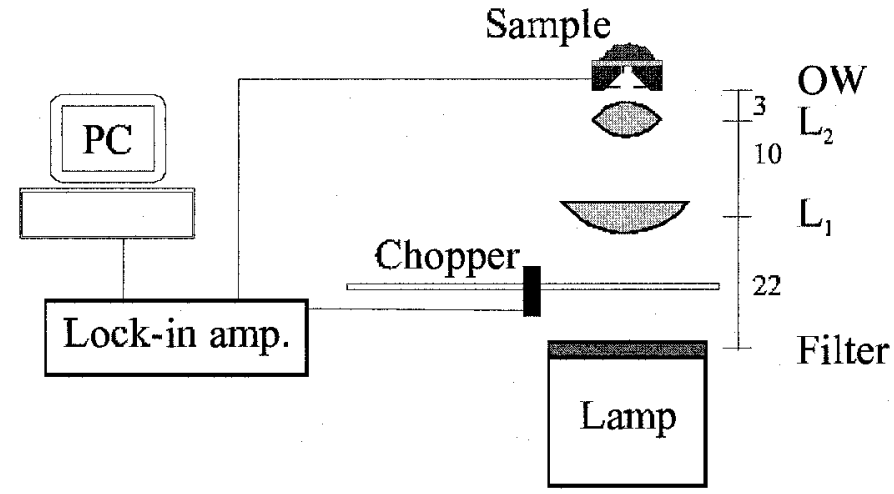

FIg. 2. Schematic view of the experimental setup (see text for the meaning of abbreviations used). The distances between the lamp, the lenses, and the $\mathrm{OW}$ are given in millimeters.

and $800 \mathrm{ppmv} \mathrm{D}_{2} \mathrm{O}$ were then used to calibrate response of the OW experimental setup. Samples of blood serum (150 to 400 ppmv $\mathrm{D}_{2} \mathrm{O}$ ) were prepared for analysis according to a method recommended in the literature that includes a vacuum sublimation step to separate water and $\mathrm{D}_{2} \mathrm{O}$ from the serum. ${ }^{8,9}$

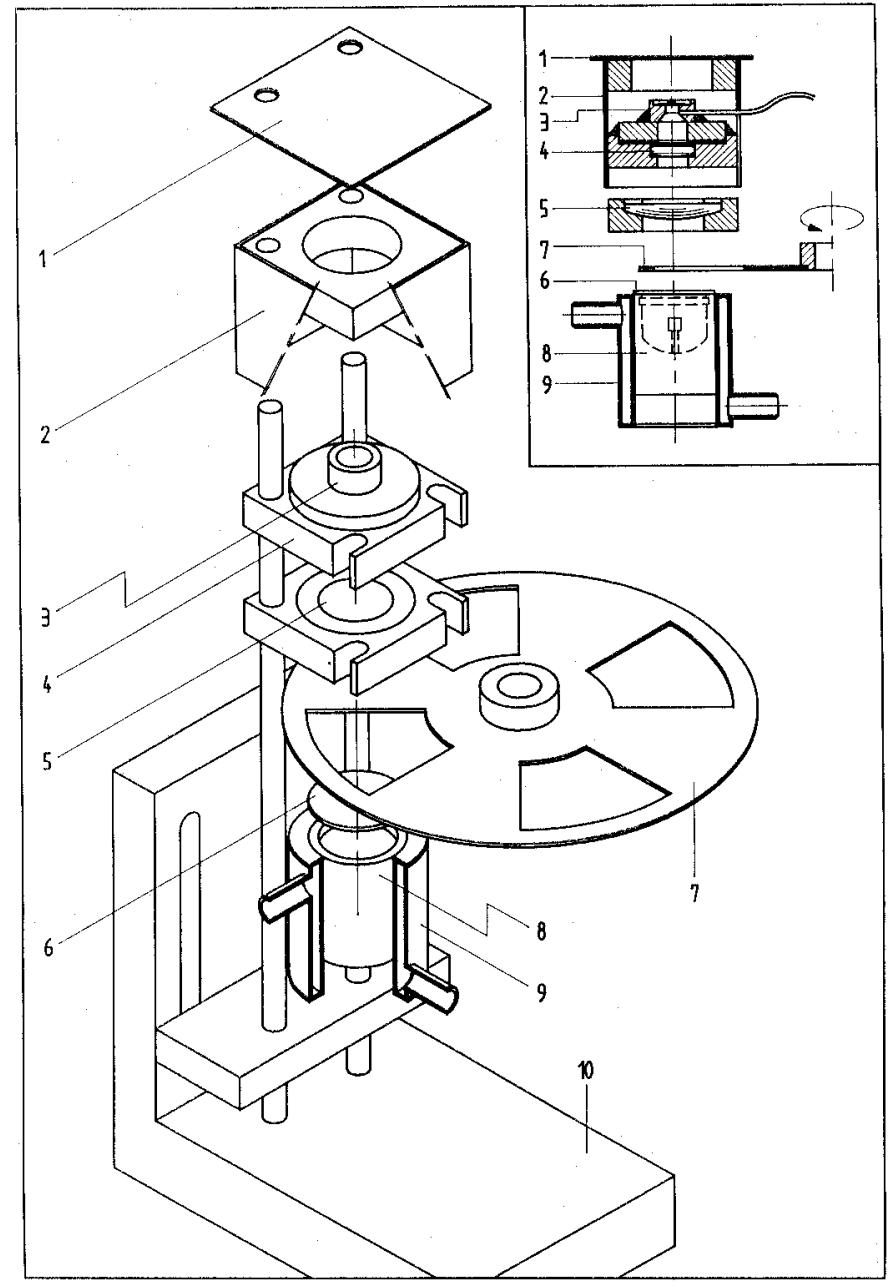

FIG. 3. Exploded view of the assembly: removable cover (1), aluminum cap (2), optothermal window (3), lens $\mathrm{L}_{2}(\mathbf{4})$, lens $\mathrm{L}_{1}(\mathbf{5})$, interference filter (6), chopper blade (7), CL 921 thermal radiation source (8), cooling jacket (9), and L-shaped carrying platform (10). 

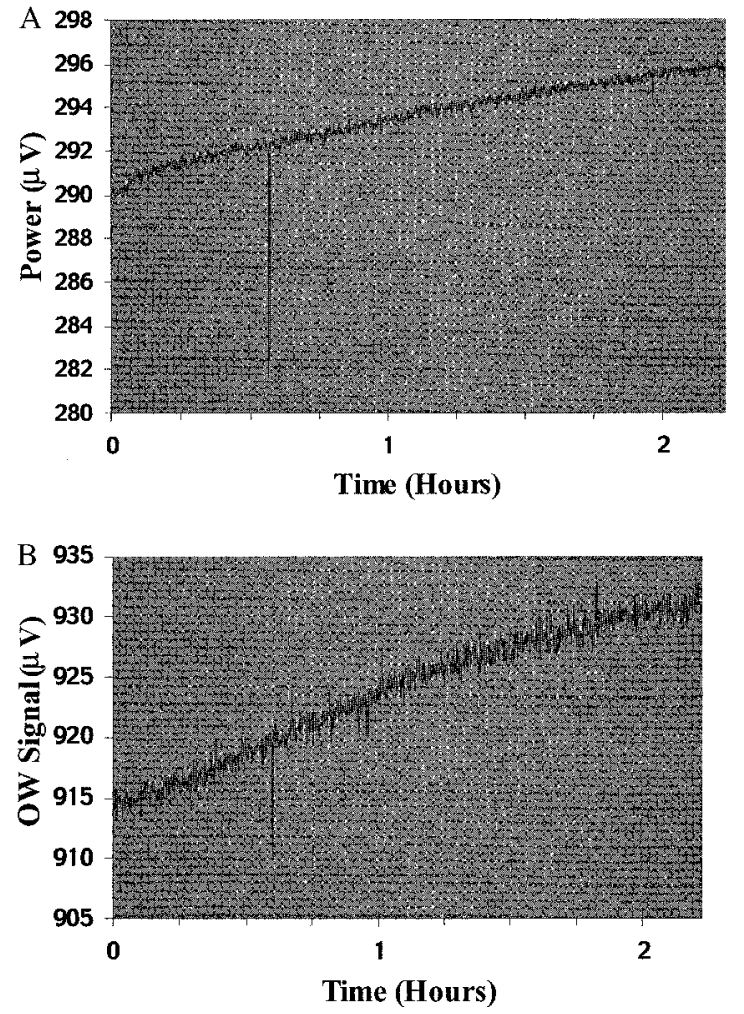

FIg. 4. (A) The power (expressed in $\mu \mathrm{V}$ ) of the CL921 lamp measured behind the empty $\mathrm{OW}$ during the first $2 \mathrm{~h}$ after the instrument has been turned on. (B) The OW signal (measured during the initial $2 \mathrm{~h}$ after the instrument has been turned on) obtained from the blank at $5 \mathrm{~Hz}$. After $2 \mathrm{~h}$, the drift in power stability drift is reduced to $2.5 \mu \mathrm{V} / \mathrm{h}$, corresponding to $60 \mathrm{ppmv} / \mathrm{h}$.

\section{MEASUREMENTS AND RESULTS}

Power stability of the radiation source is of paramount importance for reliable OW measurements. Satisfactory stability of output power of the CL921 lamp (which was maintained at $20^{\circ} \mathrm{C}$ ) could be obtained but only at the expense of a relatively long time interval needed to reach the equilibrium. Figure 4A shows time dependence of the CL921 lamp output power (expressed in microvolts) detected with the PVDF pyroelectric detector when the latter was placed above an empty OW disk; at $5 \mathrm{~Hz}$ modulation frequency the corresponding $\mathrm{OW}$ signal was 60 $\mu \mathrm{V}$. Figure 4B, obtained under identical conditions, displays the OW signal obtained from distilled water. Direct proportionality between OW signal (Fig. 4B) and excitation power (Fig. 4A) is clearly observed. Large transient peaks in Figs. 4A and $4 \mathrm{~B}$ are due to the mechanical vibrations accidentally generated in the laboratory. After $3 \mathrm{~h}$ the output power of the lamp stabilized, and the OW signal from the distilled water approached a constant level of $937 \mu \mathrm{V}$. Unfortunately, due to the present construction of the setup, it is difficult to insert a beamsplitter into the radiation beam in order to couple out a portion of the lamp power for normalization purposes. For that reason, all subsequent OW measurements were carried out at least $3 \mathrm{~h}$ after the experimental setup had been turned on.

Long time intervals needed to reach steady OW signals are due to the fact that the OW cell itself was not thermally stabilized. Since the absorption coefficient of $\mathrm{D}_{2} \mathrm{O} /$

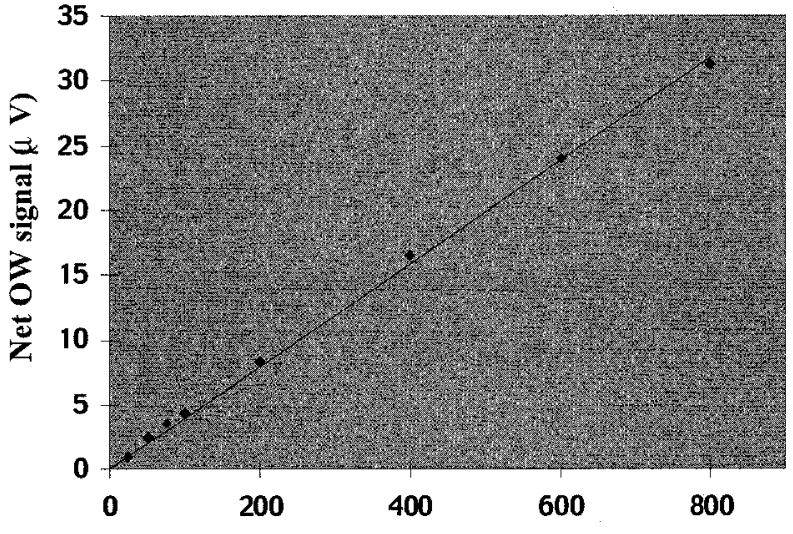

Concentration of $\mathrm{D}_{2} \mathrm{O}$ in water (ppmv)

FIG. 5. Net OW signal plotted against the concentration of the heavy water in the mixture. Each point in a diagram is the average of 200 values.

water mixtures depends strongly on temperature (for example, a temperature change of $1{ }^{\circ} \mathrm{C}$ causes a change in absorption coefficient equivalent to a $38 \mathrm{ppmv}$ error in the concentration), ${ }^{2}$ the need for temperature stabilization of the $\mathrm{OW}$ is therefore self-evident.

As a next step, OW measurements were performed with a series of standard solutions containing $25,50,75$, $100,200,400,600$, and $800 \mathrm{ppmv} \mathrm{D}_{2} \mathrm{O}$ in distilled water. Measurement with a blank (i.e., distilled water) was performed $90 \mathrm{~s}$ after it was deposited on a sapphire disk. Data for the blank were taken each half-second over 100 s (lock-in integration time $3 \mathrm{~s}$ ), resulting in 200 values for the OW signal. The blank sample was then removed by wiping it off the sapphire disk with a tissue. The complete measurement cycle (i.e., loading of the sample, waiting for $90 \mathrm{~s}$, measuring over $100 \mathrm{~s}$, and then rejecting the sample) took about $200 \mathrm{~s}$.

The OW was then loaded with the test sample containing lowest ( $25 \mathrm{ppmv}$ ) $\mathrm{D}_{2} \mathrm{O}$ concentration, and the procedure described above was repeated. In this case each of the 200 lock-in readings represents the total OW signal due to the absorption of $25 \mathrm{ppmv} \mathrm{D}_{2} \mathrm{O}$ present in the sample and a residual (1000000 ppmv-25 ppmv) absorption of water.

In order to derive the net OW signal (i.e., the OW signal due solely to the absorption of $\mathrm{D}_{2} \mathrm{O}$ ), it is necessary to subtract the blank OW signal from the total OW signals. In doing so, one first vectorially adds all 200 values obtained from the blank sample before dividing the sum by 200 to compute the average OW signal for the blank. The average signal from a blank thus obtained is then vectorially subtracted from each of the 200 total OW signals obtained from a given test sample. Repeating the same procedure using other standard solutions enables one to construct the calibration curve (Fig. 5) showing net $\mathrm{OW}$ signal as a function of $\mathrm{D}_{2} \mathrm{O}$ concentration. The analysis of data in Fig. 5 yields a 30 ppmv $\mathrm{D}_{2} \mathrm{O}$ for a limit of detection (LOD) that is equivalent to a minimum detectable absorption coefficient of $0.5 \mathrm{~cm}^{-1}$. The above-mentioned LOD was calculated by taking the ratio of a triple standard deviation from the blank OW signal and the slope of the calibration curve shown in Fig. 5.

The aim of a final experiment was to compare the per- 


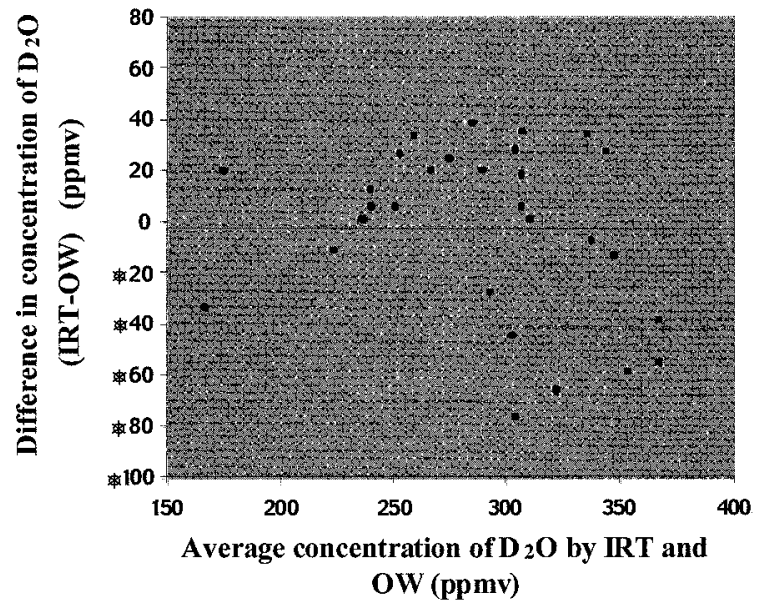

FIG. 6. Comparison of results (29 samples of blood serum were measured) obtained by the IRT and OW techniques. The upper and lower dotted lines refer to the range of a double standard deviation (on either side of the straight line $y=-3$ representing the arithmetic average of all values along the $\mathrm{y}$ axis).

formance of the OW method to that of the infrared transmission technique often used in clinical practice to measure $\mathrm{D}_{2} \mathrm{O}$. Twenty-nine samples prepared from human blood (containing $\mathrm{D}_{2} \mathrm{O}$ concentrations ranging from 150 to $400 \mathrm{ppmv}$ ) were studied. The protocol for the $\mathrm{OW}$ measurement and calculation of OW signals were the same as described above. With respect to the IRT study by means of a commercial instrument, the concentration of each specimen was measured only once.

Figure 6 shows a comparison of the two methods: the difference in concentration $c_{\mathrm{IRT}}-c_{\mathrm{OW}}$ measured by the IRT and OW methods is plotted against the arithmetic mean $\left[c_{\mathrm{IRT}}+c_{\mathrm{OW}}\right] / 2$. This widely adopted approach used to compare results obtained by two different methods was originally proposed by Bland and Altman. ${ }^{10}$ The two methods provide consistent results with data points (Fig. 6) scattered on either side of a line $y=-3$, corresponding to a mean difference. The limit of agreement defined as the range of $y$ values spanning the four standard deviations (two above and two below the line $y=-3$ ) is 76 ppmv.

\section{CONCLUSION}

The amplitude measurement of the OW signal allows one to determine the concentration of $\mathrm{D}_{2} \mathrm{O}$ in blood serum. Direct thermal contact between the sample and the disk is essential for good OW measurements. The 30 ppmv LOD obtained for the OW method in studies of standard solutions is comparable to $17 \mathrm{ppmv}$ attainable by IRT. Likewise, results of studies on human blood serum indicate a good agreement between concentration values obtained by the IRT and OW methods.

Although, at present, results of our studies suggest comparable sensitivities for both methods, pursuing the development of the OW approach is encouraged as there is room for further improvements. We believe that a compact and moderately priced $\mathrm{OW}$-based $\mathrm{D}_{2} \mathrm{O}$ instrument can be constructed, and that its performance might eventually surpass that of an IRT instrument. This possibility is important because, in that case, the OW concentration measurement will be able to be performed at a reduced $\mathrm{D}_{2} \mathrm{O}$ intake (and, hence, reduced costs) without a loss in sensitivity.

The most direct way to try to improve the performance of the present setup is to optimize the design of the $\mathrm{OW}$ itself; this approach implies the choice of proper window material and piezoelectric sensor, size of the clear disk aperture, etc. In using this approaches one is governed by the need to achieve the highest possible transmission for a disk at operating wavelength while simultaneously minimizing its thermal mass. Some preliminary calculations indicate that a factor of 2 gain in sensitivity is achievable in this manner.

Further enhancement is expected if the OW can be designed to operate at a (stabilized) temperature below that of the ambient. This is the case because for heavy water mixtures $\mathrm{d} \beta / \mathrm{d} T$ is negative; for example, performing the $\mathrm{OW}$ experiment at $5^{\circ} \mathrm{C}$ is expected to provide an OW signal twice as high as at a room temperature. Combined with the above-mentioned enhancement resulting from disk optimization, one would obtain a factor of 4 overall improvement.

The most important consideration, however, is to get as much power as possible to reach the sample. A drawback of the present setup is the relatively low amount of excitation power mainly due to the intrinsically low power emitted at $4 \mu \mathrm{m}$ by the CL921 thermal source and the use of optical components that all introduce optical losses. To avoid this problem, it is recommended that one use a gold-coated cone the top of which is brought in proximity to the sapphire disk. An alternative to improve the efficiency in collection of the lamp radiation is to insert a highly transparent fiber (for example, silver halide) between the source and the disk. Although without performing the actual experiment it is difficult to predict exactly how much can be gained, we believe that improvement by a factor between 2 and 3 is likely. All this means is that, by paying more attention to the choice of materials used to construct the OW, by stabilizing the temperature of the OW, and by more efficiently collecting the power emitted by the lamp, one can anticipate one order of magnitude gain in sensitivity for the present setup.

More can be gained if the lamp is replaced by a more intense $4 \mu \mathrm{m}$ radiation source providing a good-quality beam. Since in such a case the interference filter and lenses are no longer needed, one reasons that practically all the power emitted by the source will also reach the sample. Novel powerful and stable sources have already emerged (e.g., a color center laser and overtone CO laser) or are still under development (a compact-size diode laser). Most likely, additional factor of 10 gains in sensitivity could be obtained, but at the present stage of technology this can be achieved only with additional expense.

In general, the new $\mathrm{OW}$ design should provide the possibility for the normalization of lock-in signals, thereby enabling one to carry out measurements immediately after turning on the source. Since the $\mathrm{OW}$ is prone to mechanical and acoustic vibrations, care must be exercised to isolate it from the surrounding equipment and other sources of disturbance. As far as the electronic equipment needed to detect $\mathrm{OW}$ signal is concerned, considerable cost savings may be anticipated, since only a simplified 
version of the lock-in amplifier operating at a single modulation frequency is required.

Finally, although all measurements described in this paper were carried out in the "static" regime (that is to say, with the sample deposited on a thin disk), it is relatively simple to design a flow-through OW-based device that, in conjunction with the autoanalyzer, can be employed for numerous on-line applications; work on this approach is already in progress.

In conclusion, the $\mathrm{OW}$ measurement of $\mathrm{D}_{2} \mathrm{O}$ is characterized by reasonable sensitivity, good reproducibility, and ease of sampling (loading the sample and cleaning the disk). These feature combined with the compact size of the OW device, the low tracer intake, and the moderate investment cost make the $\mathrm{OW}$ a simple and promising method for the determination of $\mathrm{D}_{2} \mathrm{O}$ and, hence, for the assessment of body composition in, for example, studies of obesity and pursuit of community-scale surveys.

\section{ACKNOWLEDGMENTS}

We thank Mr. Iwan Koenderman and Dr. Jan Paul Favier for their pioneering work in the field of OW; the technical staff for their rapid and enthusiastic support; Mr. Mees Schimmel for providing some of the illustrations; and Dr. Paul Duerenberger (Department of Human Nutrition and Epidemiology), Mr. Cees van Asselt, electronic engineer, and Prof. Mihai Chirtoc (Institute of Isotopes and Molecular Technology) for much valuable advice received in the course of this study. This research study was carried out within the framework of the INCOCopernicus project (Contract Number ICICT961003) funded by the DGXII of the European Community in Brussels, Belgium.

1. G. Bedogni, M. R. Bollea, S. Severi, O. Trunfio, A. M. Mauzieri, and N. Battistini, Eur. J. Clin. Nutr. 51, 129 (1997).

2. H. C. Lukaski and P. E. Johnson, Am. J. Clin. Nutr. 41, 363 (1985).

3. R. Hanna, C. H. Long, R. Y. Asfour, R. N. Lutz, and S. E. Zobrisky, in Body Composition in Animal and Men, Proceedings of Symposium held at the University of Missouri, Columbia, Publication 1598 (National Academy of Sciences, Washington, D.C., 1968), pp. $480-491$.

4. G. M. Halle and M. R. Querry, Appl. Opt. 12, 555 (1979).

5. E. H. Braue and M. G. Panella, Appl. Spectrosc. 41, 1213 (1987).

6. P. Helander, J. Appl. Phys. 59, 3339 (1986).

7. J. P. Favier, Ph.D. Thesis, Wageningen University and Research Center, Wageningen, The Netherlands (1987).

8. M. J. Stansell and L. Mojica, Jr., Clin. Chem. 14, 1112 (1968).

9. P. R. Schloerb, B. J. Friis-Hansen, I. S. Edelman, and F. Moore, J. Clin. Invest. 29, 1296 (1950).

10. M. J. Bland and D. G. Altman, Lancet 1, 307 (1986). 